\title{
High-performance Sn@carbon nanocomposite anode for lithium-ion batteries: Lithium storage processes characterization and low-temperature behavior
}

\author{
F. Nobili ${ }^{\mathrm{a}, *}$, I. Meschini ${ }^{\mathrm{b}}$, M. Mancini ${ }^{\mathrm{a}}$, R. Tossici ${ }^{\mathrm{a}}$, R. Marassi ${ }^{\mathrm{a}}$, F. Croce ${ }^{\mathrm{b}, * *}$ \\ a Scuola di Scienze e Tecnologie, Sezione Chimica, Università di Camerino, Via S. Agostino 1, I-62032 Camerino, Italy \\ b Dipartimento di Farmacia, Università “G. D’Annunzio” Chieti-Pescara, Via dei Vestini 31, I-66100 Chieti, Italy
}

\section{A R T I C L E I N F O}

\section{Article history:}

Received 4 May 2013

Received in revised form 27 May 2013

Accepted 30 May 2013

Available online 18 June 2013

\section{Keywords:}

Li-ion battery

$\mathrm{Sn}-\mathrm{C}$ anode material

Nanocomposite electrode

Electrospinning

\begin{abstract}
A B S T R A C T
The electrochemical behavior of a composite anode for Li-ion batteries, based on nanosize tin particles embedded in electrically conducting porous multichannel carbon microtubes (Sn-PMCMT), synthesized by co-electrospinning, is here evaluated.

An activation protocol aimed at maximizing anode mechanical stability and capacity retention upon cycling is presented. The results are compared with those obtained with an anode using pristine PMCMT carbon as active material.

The Li uptake and release processes by $\mathrm{Sn}$ and $\mathrm{C}$ are evaluated by galvanostatic charge/discharge cycles, in order to differentiate the two contributions to the overall anode capacity. Electrochemical impedance spectroscopy (EIS) analysis is utilized in order to evaluate possible improvements to chargetransfer kinetics due to the nanosize Sn particles dispersion. Finally, the performances of the composite Sn-PMCMT anode are characterized at different charge/discharge currents and temperatures.

The anode can deliver a capacity of $500 \mathrm{mAh} \mathrm{g}^{-1}$ for more than 300 cycles, most of them at $1 \mathrm{C}$ or higher charge/discharge rate, which confirms its very high, stable cycling performances. Moreover, this tailored nanostructured anode retains a relevant amount of capacity even in very demanding cycling conditions, as the case for very low temperatures. These results make the proposed Sn-PMCMT an ideal candidate anode for high-performance Li-ion batteries able to operate in a wide array of operating conditions.
\end{abstract}

(c) 2013 Elsevier Ltd. All rights reserved.

\section{Introduction}

Nowadays, modern society is facing challenging energy and environment issues, which, undoubtedly, will require the substitution of fossil fuels with greener energy sources for electric energy generation and ground transportation. Renewable energy sources are the most suited option to this purpose, provided efficient devices for electric energy storage and delivery are developed. At the moment, due to their versatility and scalability, lithium-ion batteries represent the best powering opportunity both for portable electronics and for transportation. In fact, the alternative fuel cell based technology, at the moment, is not sufficiently mature as power source for electric vehicles, mainly due to operational factors related to electro-catalysis and to gas feeding and storage [1].

\footnotetext{
* Corresponding author. Tel.: +39 0737 402210; fax: +39 0737402296.

** Corresponding author. Tel.: +39 0871 3554480; fax: +39 08713554483.

E-mail addresses: francesco.nobili@unicam.it (F. Nobili), fausto.croce@unich.it (F. Croce).
}

In order to satisfy the high power and energy densities requirements mandatory for ground transportation applications, new chemistries other than 'conventional' anode and cathode materials, currently based on graphite and lithium oxides, are necessary [2,3]. In this context, great expectations are posed on high-capacity anode materials that rely on alloying reactions. Tin is the element that has attracted great attention, as it can be reversibly lithiated up to the end compound $\mathrm{Li}_{4.4} \mathrm{Sn}$ with a theoretical reversible capacity of $994 \mathrm{mAh}^{-1}$. Despite its very high capacity, the practical use of Sn anodes has been hindered by the dramatic large volume expansion of the unit cell - which reaches $300 \%$ of its initial value - associated with the reversible lithium alloying and de-alloying during the battery operation. As a consequence, the active material particles are subject to a severe mechanical stress that is the main cause of electrode failure upon cycling. Another drawback often associated with alloying anodes is the high irreversible capacity exhibited during the initial cell cyclations caused by lithium-sequestration irreversible processes such as SEI formation on tin and carbon particles [4-9], being these latter always present in the composite electrode formulation in order to guarantee a sufficient electronic conductivity above the electron percolation threshold [10]. To such 
respect, engineered electrode morphologies at nano-scale could reduce the large volume variation of tin particles and increase the reversibility of charge-discharge process. Common strategies adopted to tackle these issues involve either the thoroughly intermixing (dispersion) of Sn particles with graphite or amorphous carbon, or Sn particles encapsulation into optimized carbon nanostructures such as mesoporous or nano-tubular [11,12].

In a recent paper [13], we have introduced a $\mathrm{Sn} / \mathrm{C}$ composite anode in which metal tin is embedded in electrically conducting porous multi-channel carbon microtubes (Sn-PMCMT). We have demonstrated that this kind of electrode is able to deliver high capacities at charge-discharge rates ranging from $0.7 \mathrm{C}$ to $10 \mathrm{C}$. Nevertheless, it has been shown that Sn-PMCMT electrodes still suffer from a capacity fading on long cyclations even if the Li-Sn alloy volume changes are buffered by the peculiar carbon microstructure.

In the present paper we report on an optimized cycling protocol aimed at increasing the reversibility of Sn-PMCMT anodes. In addition, in order to separate the tin and the carbon contributions to the overall anode delivered capacity, the electrochemical behavior of the Sn-PMCMT anode is compared to that of a composite anode comprising only the multi-channel-micro-tubular(PMCMT) carbon particles. Finally, the performances of anodes based on Sn-PMCMT are analyzed in a wide temperature and rate range in order to evaluate their potentialities in more demanding applications.

\section{Experimental}

The Sn-PMCMT material has been prepared according to a wellestablished procedure reported in Ref. [13]. Briefly, a mixture of precursors (PAN, PMMA, tributylphenyl-Sn) has been electrospun onto an $\mathrm{Al}$ foil by applying a $\mathrm{kV}$ range voltage, then stabilized in air at $250^{\circ} \mathrm{C}$ and finally pyrolyzed under $\mathrm{Ar} / \mathrm{H}_{2}$ atmosphere at $700^{\circ} \mathrm{C}$. After cooling, the Sn-PMCMT mat has been ground in an agate mortar in order to obtain a powder micrometer sized. An analogous approach has been followed for the synthesis of pristine PMCMT fibers without any encapsulated Sn, by using toluene in substitution of tributylphenyl-Sn precursor. In this latter case, the presence of toluene, as a substitute of tributylphenyl-Sn, assures both comparable rheology and polarity to the polymeric solution. The solution of PAN, PMMA and toluene was analogously electrospun onto an $\mathrm{Al}$ foil and thermally treated at $250^{\circ} \mathrm{C}$ and $700^{\circ} \mathrm{C}$ in the same experimental conditions adopted for the synthesis of Sn-PMCMT.

The morphology of the synthesized fibers was investigated using scanning electron microscopy (SEM, Mod. EVO 50 - Zeiss) in order to gain information on the shape and width of the fibers.

Electrodes of both materials have been manufactured by preparing slurries containing active material (either Sn-PMCMT or PMCMT):Super-P:binder/85:5:10 (mass ratio) dissolved in highpurity deionized water. The use of an aqueous solution of $\mathrm{Na}$-carboxymethylcellulose (Na-CMC by Aldrich, MW $\sim 250,000$ ) as binder ensured a 'green' electrode fabrication process, which has been reported to guarantee stable interfacial and mechanical properties [13-17]. The slurries were spread onto $\mathrm{Cu}$ foils using the doctor-blade technique. Circular electrodes of $9 \mathrm{~mm}$ diameter were cut from these latter foils and then dried at $120^{\circ} \mathrm{C}$ under vacuum. The active mass loadings resulted of about $1 \mathrm{mg} \mathrm{cm}^{-2}$ for both anode materials.

Electrochemical test cells (Swagelock type T-cells) were assembled in an Ar-filled glove box (Mod. UniLab-MBraun, oxygen and water content below $1 \mathrm{ppm}$ ), using Li metal disks as counter and reference electrodes. A Whatman glass fiber (thickness $=300 \mu \mathrm{m}$ ) was used as separator, while a $1 \mathrm{M}$ solution of $\mathrm{LiPF}_{6}$ in EC:DEC:DMC ternary solvent mixture (LP71 by Merck) was used as electrolyte. This solvent is optimized for low-temperature electrochemical behavior by preventing solvent freezing and giving an acceptable $\mathrm{Li}^{+}$mobility $[18,19]$.
The electrochemical characteristics of the cells have been investigated at selected temperatures between $20^{\circ} \mathrm{C}$ and $-20^{\circ} \mathrm{C}$; after each temperature change, the cells have been allowed to equilibrate for $5 \mathrm{~h}$ before running the electrochemical measurements. All the potentials are referred to the $\mathrm{Li}^{+} / \mathrm{Li}$ redox couple.

\section{Results and discussion}

\subsection{Chemical and morphological characterization}

The characterization of the Sn-PMCMT composite anode material is well detailed in Ref. [13]. Briefly, most of Sn is dispersed through the hollow nano-channels that build up the carbon microfibers. The approximate width of the carbon microfibers is about $1 \mu \mathrm{m}$, while the average size of dispersed Sn grains is below $100 \mathrm{~nm}$. In addition, minor amounts of carbon are present as grains of irregular shapes and micrometer size, through which the Sn is still dispersed at nanosized level. Elemental analysis and X-ray diffraction phase refinement revealed a Sn:PMCMT mass ratio of 33.3:66.7. Most of the $\mathrm{Sn}$ is present as metal and minor amounts as oxides, while PMCMT contains, in addition to $\mathrm{C}$, some $\mathrm{H}$ and $\mathrm{N}$ impurities from organic precursors. Fig. 1 shows a SEM micrograph of the bare PMCMT matrix synthesized as described in Section 2. Also in this case, the material is made up by intimately tangled fibers of carbon, about $1 \mu \mathrm{m}$ wide. This confirms that the synthetic route adopted successfully replicated the carbon fibers morphology of Sn-PMCMT sample. Elemental analysis confirmed that most of the PMCMT powder is made of $\mathrm{C}$, while $\mathrm{H}$ and $\mathrm{N}$ globally account for about $15 \%$ (mass ratio), as residuals of the thermal treatment of the organic precursors.

\subsection{Capacity-limited charge/discharge behavior of Sn-PMCMT} anode

Sn-PMCMT anodes underwent galvanostatic charge/discharge cyclations in the potential range $0.01 \mathrm{~V}<E<2 \mathrm{~V}$ and at specific currents of $700 \mathrm{mAg}^{-1}$ (cycles 1-100) and $500 \mathrm{mAg}^{-1}$ (cycles 101-200). When the cell was discharged (corresponding to Sn-PMCMT lithiation) the electrode was charge-limited in order to minimize the large volume expansion and contraction processes, which usually accompany the initial cycles of Li-Sn alloying/dealloying and lead to progressive mechanical failure of the tin particles and loss of performance. In fact, during the activation cycles a large amount of charge is usually consumed for irreversible Li storage processes (formation of solid electrolyte interphase, SEI

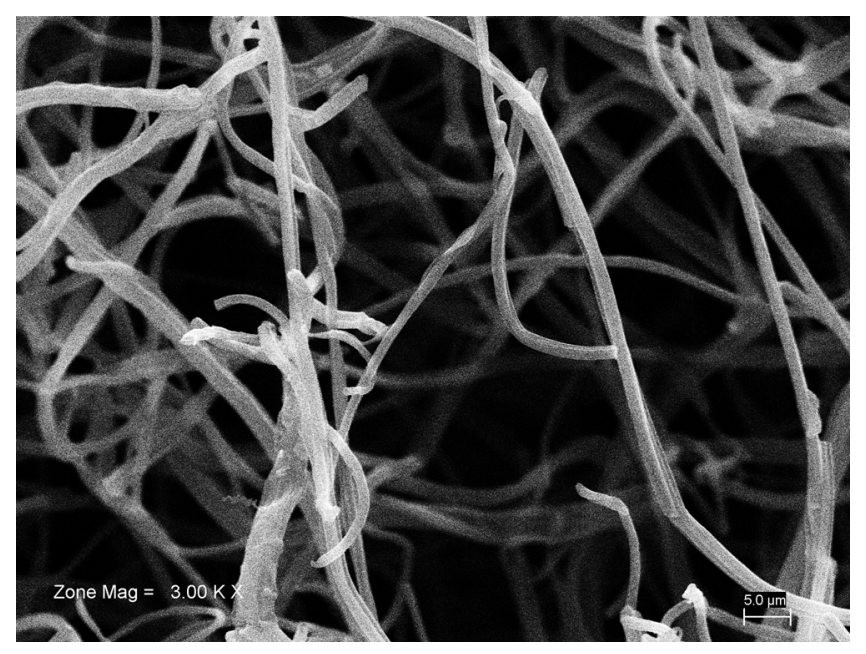

Fig. 1. SEM micrograph of PMCMT matrix. 


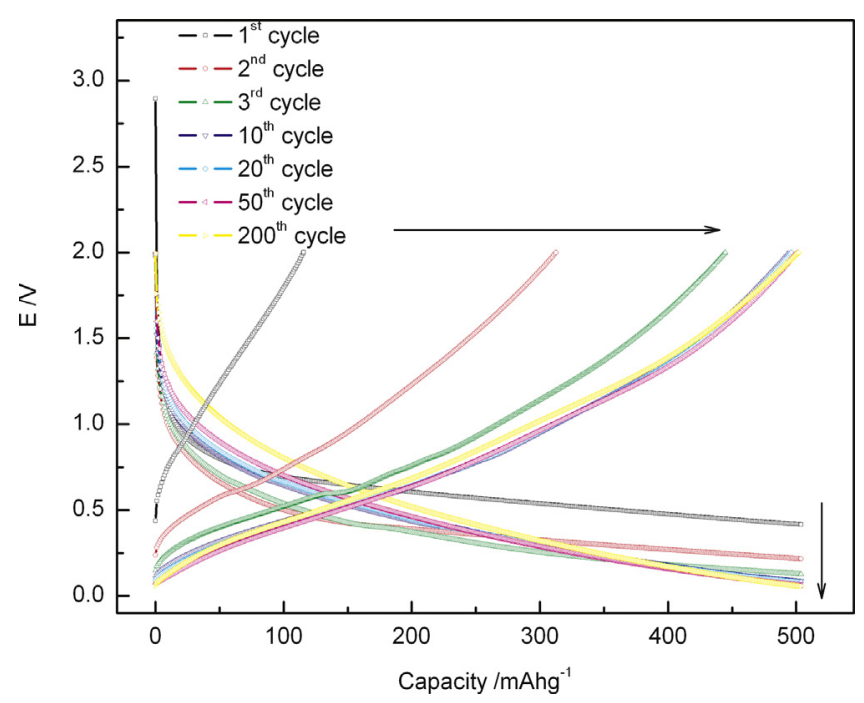

Fig. 2. Charge/discharge profiles of Sn-PMCMT anode. $T=20^{\circ} \mathrm{C}$. Limiting capacity $=500 \mathrm{mAh} \mathrm{g}^{-1}$; specific currents $=700 \mathrm{mAg}^{-1}$ (cycles $1,2,3,10,20$, and 50), $500 \mathrm{mAg}^{-1}$ (cycle 200).

[4-7]; accumulation of Li at defects and nano-voids of carbon surface $[12,20])$. Therefore, if the discharge capacity is limited we can expect that during the first cycle most of the exchanged Li is involved in irreversible processes and that only minor amounts are available for reversible storage in Li-Sn alloys, so that the volume expansion of active $\mathrm{Sn}$ is limited. As the charge/discharge cycles proceed, the irreversible capacity is expected to progressively reduce, the $\mathrm{Sn}$ utilization to increase and the $\mathrm{Li}-\mathrm{Sn}$ volume variations to become larger. This implies that the mechanical stresses associated with volume expansion and contraction may be 'distributed' over several cycles, supposedly leading to improved electrode stability.

The limiting capacity was set to $500 \mathrm{mAhg}^{-1}$, which is the value of the reversible capacity commonly expected for analogous $\mathrm{Sn} / \mathrm{C}$ composite electrodes [21-25]. As a consequence, the applied $700 \mathrm{mAg}^{-1}$ and $500 \mathrm{mAg}^{-1}$ specific currents correspond to C-rate values of $1.4 \mathrm{C}$ and $1 \mathrm{C}$, respectively, in agreement with Ref. [13].

Fig. 2 shows the galvanostatic charge/discharge profiles of some relevant cycles. As expected, during the first discharge most of the $500 \mathrm{mAhg}^{-1}$ limiting capacity is used for irreversible Li-C processes (SEI formation, $\mathrm{Li}$ accumulation at $\mathrm{C}$ pores and cavities), while only a minor amount remains available for reversible $\mathrm{Li}-\mathrm{Sn}$ alloying and $\mathrm{Li}-\mathrm{C}$ staging. This implies that the discharge is interrupted at about $0.4 \mathrm{~V}$, which corresponds to an alloy stoichiometry of about $\mathrm{Li}_{2.5} \mathrm{Sn}$ [26]. However, due to polarization induced by kinetic issues, the coexistence of several Li-Sn phases may be expected. During the subsequent charge, only the lithium reversibly inserted is extracted from the electrode and contributes to capacity. As a consequence, the first charge capacity results limited to $100 \mathrm{mAhg}^{-1}$. This confirms that during the previous discharge almost $400 \mathrm{mAhg}^{-1}$ have been used for irreversible $\mathrm{Li}-\mathrm{C}$ processes, resulting in a ratio irreversible/reversible capacity of about $4: 1$. As the cyclations proceed, the irreversible processes become less important, higher amounts of Li are reversibly exchanged, the Li content in Li-Sn alloys is increased and lower cutoff potentials are approached when the $500 \mathrm{mAh} \mathrm{g}^{-1}$ capacity limit is reached. The trend of charge/discharge capacity as a function of cycle number is shown in Fig. 3. The inset shows the discharge cutoff potential and the Coulombic efficiency obtained during the first 20 cycles. After 10 cycles the Coulombic efficiency tends to unity, implying that almost the whole capacity is exchanged for reversible processes, while a cut-off potential of about $0.08 \mathrm{~V}$ is reached. This

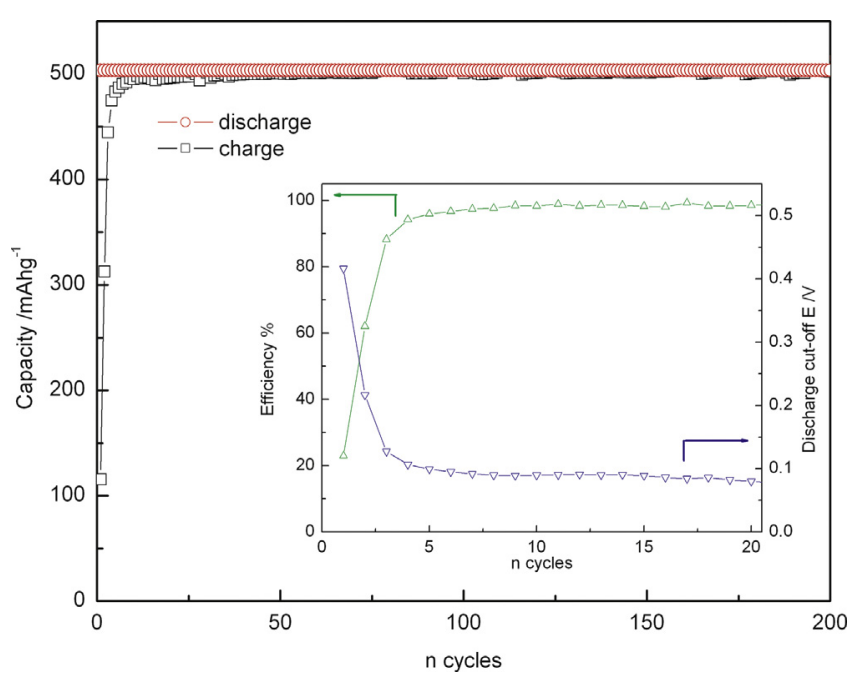

Fig. 3. Charge/discharge capacities of Sn-PMCMT anode. $T=20^{\circ} \mathrm{C}$. Limiting capacity $=500 \mathrm{mAh} \mathrm{g}^{-1}$; specific currents $=700 \mathrm{mAg}^{-1}$ (cycles $1-100$ ), $500 \mathrm{mAg}^{-1}$ (cycles 101-200). The inset shows Coulombic efficiency and the cut-off potential reached at the end of discharge for cycles 1-20.

suggests that both $\mathrm{Li}-\mathrm{Sn}$ and $\mathrm{Li}-\mathrm{C}$ processes now contribute to $\mathrm{Li}$ reversible storage $[12,23,24,26,27]$. After the initial cycles, the cell is able to reversibly deliver the requested capacity of $500 \mathrm{mAh} \mathrm{g}^{-1}$ for 200 cycles (1-100 at 1.4C, 101-200 at 1C), with Coulombic efficiency close to unity. It is thus confirmed that the nanostructured carbon microtubes are able to guarantee a stable Sn encapsulation and to prevent electrode failure, and that the cycling conditions adopted, in which Li uptake capacity is limited to $500 \mathrm{mAh} \mathrm{g}^{-1}$, contribute to buffer the volume variations of $\mathrm{Li}-\mathrm{Sn}$ alloys during the initial 'activation' cycles.

Fig. 4 shows the $\mathrm{d} Q \mathrm{~d} E^{-1}$ vs. $E$ differential profiles corresponding to the charge-discharge cycles shown in Fig. 2. These profiles confirm that during the first cycle most of the Li is irreversibly stored during discharge, while during the subsequent charge only a weak and flat electrochemical signal due to reversible release of minor amounts of Li from C matrix is revealed. The discharge profiles of the second and third cycles reveal additional irreversible Li storage processes at progressively lower potentials, occurring together with reversible $\mathrm{Li}-\mathrm{C}$ and $\mathrm{Li}-\mathrm{Sn}$ storage, as evidenced by the charge

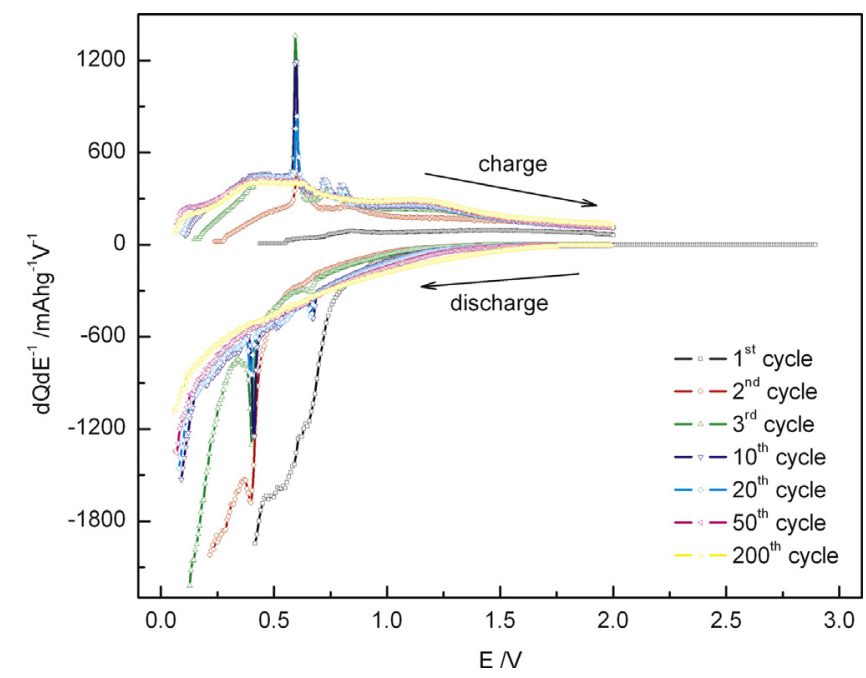

Fig. 4. Calculated $\mathrm{d} Q \mathrm{~d} E^{-1}$ vs. $E$ differential profiles of Sn-PMCMT anode. $T=20^{\circ} \mathrm{C}$; limiting capacity $=500 \mathrm{mAh}^{-1}$; specific currents $=700 \mathrm{mAg}^{-1}$ (cycles 1, 2, 3, 10, 20, and 50 ), $500 \mathrm{mAg}^{-1}$ (cycle 200). 


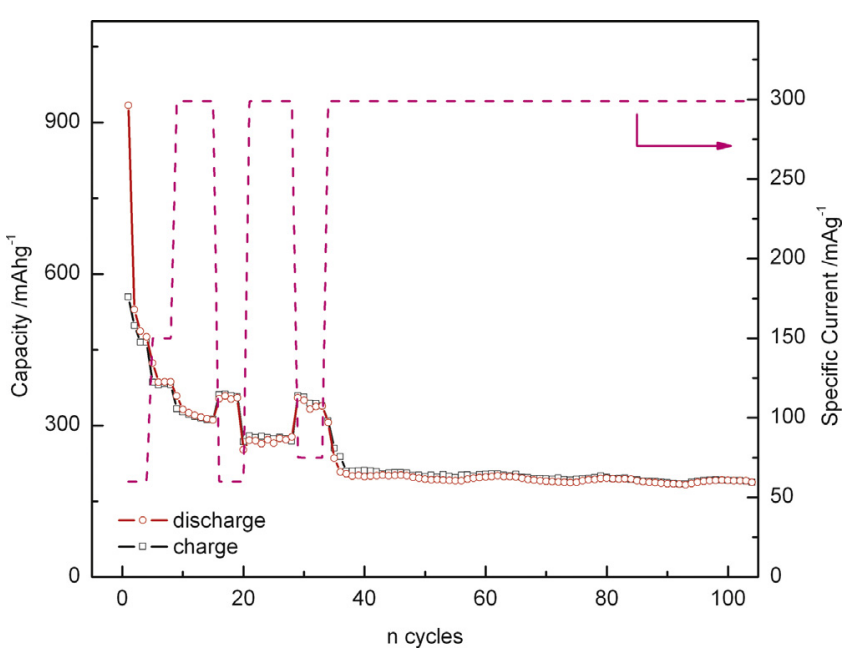

Fig. 5. Charge/discharge capacities and applied specific current of pristine PMCMT anode. $T=20^{\circ} \mathrm{C} ; 0.01 \mathrm{~V}<E<2 \mathrm{~V}$; specific currents $=60 \mathrm{mAg}^{-1}$ (cycles $1-4,16-20$ ), $75 \mathrm{mAg}^{-1}$ (cycles 29-33), $150 \mathrm{mAg}^{-1}$ (cycles 5-8), $300 \mathrm{mAg}^{-1}$ (cycles 9-15, 21-28, and 34-100).

profiles. In fact, $\mathrm{Li}-\mathrm{Sn}$ peaks in the potential region $0.45-0.9 \mathrm{~V}$ and the broad feature about $1.2 \mathrm{~V}$ (commonly related with kinetically hindered Li release from carbon $[13,20]$ ) become progressively higher (the nature of this signal will be discussed later).

During the subsequent cycles, only reversible Li uptake and release from $\mathrm{Sn}$ and $\mathrm{C}$ are evidenced, confirming the results shown in Fig. 3, in which the irreversible processes progressively fade during the initial cycles. As the cyclations proceeds, the peaks related to $\mathrm{Li}-\mathrm{Sn}$ processes tend to be hidden probably because of a progressive Sn amorphization and a sluggish kinetics. The small hump evidenced during Li release at around $0.1 \mathrm{~V}$ for the cycles 50 and 200 reveals that the contribution of $\mathrm{Li}-\mathrm{C}$ reversible processes to charge/discharge capacity progressively becomes more important.

\subsection{Charge-discharge behavior of PMCMT anode}

In order to separate the contributions to the capacity due either to $\mathrm{Li}-\mathrm{Sn}$ or to $\mathrm{Li}-\mathrm{C}$ reversible processes, the electrochemical behavior of an electrode made of pristine PMCMT carbon fibers as active material has been evaluated. A preliminary characterization of the charge/discharge behavior has been performed by galvanostatic cycles at different current densities. No capacity limitation has been set and the electrode has been left to fully charge and discharge. In these conditions it can be estimated an approximate value of maximum deliverable capacity and C-rate values can be defined: with the aim to calculate and set a limit to capacity and perform charge/discharge cycles in conditions similar to those adopted for Sn-PMCMT.

Fig. 5 shows the electrochemical performance of pristine PMCMT anode at several specific currents in the range $60-300 \mathrm{~mA} \mathrm{~g}^{-1}$. The irreversible capacity of the first cycle is about $400 \mathrm{mAh} \mathrm{g}^{-1}$, corresponding to $80 \%$ of the initial reversible capacity. After the initial cycles, the capacity rapidly drops to the values of $450 \mathrm{mAh} \mathrm{g}^{-1}$ and $300 \mathrm{mAh} \mathrm{g}^{-1}$ when specific currents of $60 \mathrm{mAg}^{-1}$ and $300 \mathrm{mAg}^{-1}$ are imposed. On continuous cycling, part of the initial capacity can be recovered if the specific current is decreased, while a steady value of about $200 \mathrm{mAh}^{-1}$ is obtained at a current density of $300 \mathrm{mAg}^{-1}$. As a consequence, the 'useful' capacity of the anode has been estimated to be in the range $200-250 \mathrm{mAh} \mathrm{g}^{-1}$. These latter values have been used to perform galvanostatic cycles with capacity limitation, in a similar way it has been done for the Sn-PMCMT anode. This in principle allows a direct, even if rough, comparison between the electrochemical behavior of the

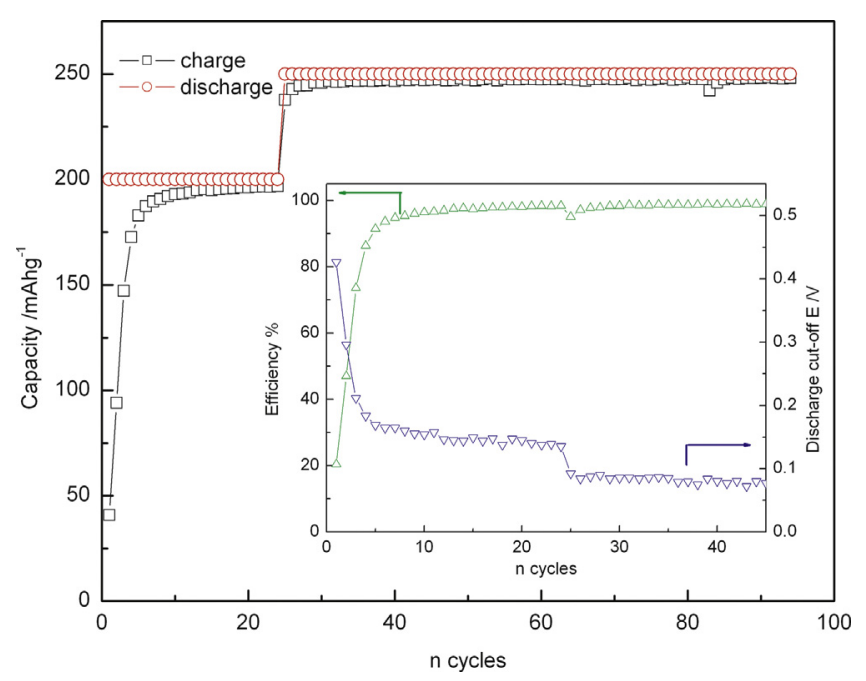

Fig. 6. Charge/discharge capacities of pristine PMCMT anode. $T=20^{\circ} \mathrm{C}$; limiting capacity $=200 \mathrm{mAh} \mathrm{g}^{-1}$ (cycles 1-24), $250 \mathrm{mAh} \mathrm{g}^{-1}$ (cycles 25-94); specific currents $=200 \mathrm{mAg}^{-1}$. The inset shows Coulombic efficiency and the cut-off potential reached at the end of discharge for cycles 1-45.

two electrodes and makes it possible to eventually separate the contributions to the Li storage from both the Sn nano-particles and the $C$ porous matrix.

Fig. 6 shows the charge/discharge behavior of PMCMT anode when limited in capacity. Initially (cycles 1-24) a value of $200 \mathrm{mAh} \mathrm{g}^{-1}$ was set as limiting capacity and a charge/discharge specific current of $200 \mathrm{mAg}^{-1}$ was applied. Analogously to Sn-PMCMT anode, during the initial cycles most of the capacity is consumed for irreversible processes. In this case, the channels of the carbon microfibers do not contain the Sn nano-particles and, as a consequence, a larger surface area is exposed to the electrolyte solution. It follows that it is reasonable to assume that almost the whole irreversible capacity can be attributed to SEI formation. After few cycles, the relative amount of irreversible capacity decreases, while there is a larger contribution from reversible capacity, due to reversible $\mathrm{Li}$ intercalation between the planes of the amorphous carbon and at the surface of carbon defects or pores $[11,12,20,27,28]$. It is worth noting that in these conditions, where the capacity is limited to $200 \mathrm{mAh} \mathrm{g}^{-1}$, the anode can reach a cut-off potential of about $0.14 \mathrm{~V}$, a value that is higher than that reached by the $\mathrm{Sn-PMCMT}$ anode $(0.08 \mathrm{~V})$ when its discharge was limited to the estimated value of 'useful' deliverable capacity (Section 3.2). This implies a lower carbon utilization for the PMCMT anode. To cope with this problem, the limiting capacity has been increased to $250 \mathrm{mAh} \mathrm{g}^{-1}$ starting from cycle 25 . Concurrently, the charge/discharge specific current has been raised to $250 \mathrm{~mA} \mathrm{~g}^{-1}$ : a value assumed, for all the following cycles, as the approximate 'unitary' $1 \mathrm{C}$ charge/discharge rate.

As a consequence of deeper anode utilization, further irreversibility is evidenced during cycles 25-30, because of the occurrence of irreversible processes at the lower accessed potentials; after that, a Coulombic efficiency close to $100 \%$ is recovered, meaning that the whole capacity is used for reversible processes. A cut-off potential of about $0.08 \mathrm{~V}$ is reached, implying that the degree of utilization of the carbonaceous matrix is now coherent with Sn-PMCMT anode. It is clear that, even for the pristine PMCMT anode, the limitation of the deliverable capacity can lead to more stable performance over time.

The charge/discharge $E$ vs. $Q$ curves shown in Fig. 7 confirm the occurrence of Li uptake and release mechanisms typical of an amorphous carbon. A hump, describing the formation of a passivation layer, can be detected at about $0.9 \mathrm{~V}$ during the first $\mathrm{Li}$ 


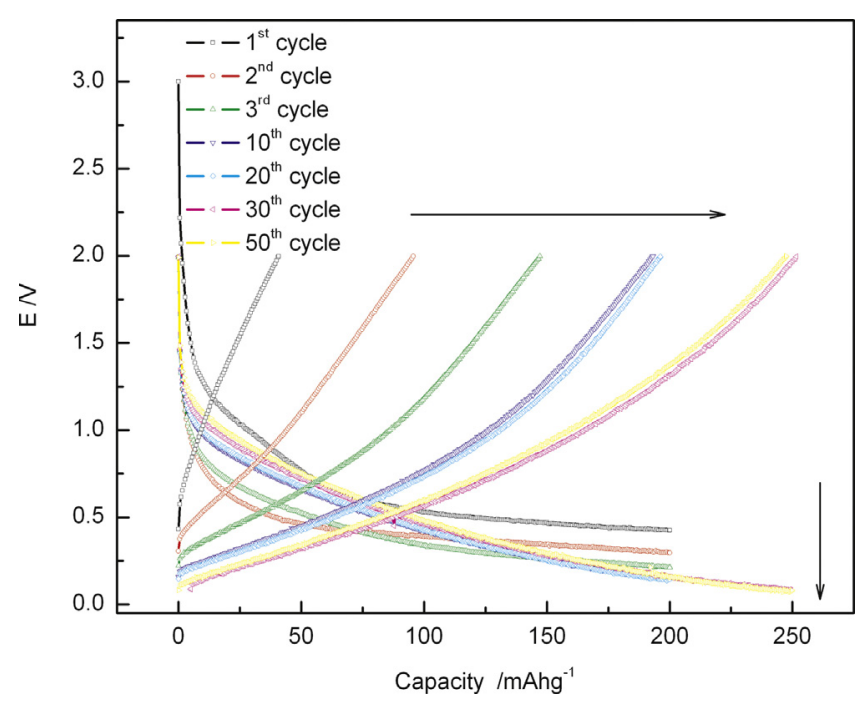

Fig. 7. Charge/discharge profiles of pristine PMCMT anode. $T=20^{\circ} \mathrm{C}$; limiting capacity $=200 \mathrm{mAh} \mathrm{g}^{-1}$ (cycles 1, 2, 3, 10, and 20), $250 \mathrm{mAh} \mathrm{g}^{-1}$ (cycles 30 and 50); specific currents $=200 \mathrm{mAg}^{-1}$.

insertion, followed by a plateau at about $0.45 \mathrm{~V}$, which describes Li storage by carbon matrix (mainly irreversible). During the first Li de-insertion, only minor amounts of $\mathrm{Li}$ are reversibly released from the amorphous carbon. During second and third Li insertions, further amounts of $\mathrm{Li}$ are irreversibly bound to the carbon matrix. This is confirmed by the differential $\mathrm{d} Q \mathrm{~d} E^{-1}$ profiles shown in Fig. 8, where pronounced shoulders are evidenced at potentials of $0.35 \mathrm{~V}$ and $0.24 \mathrm{~V}$, respectively. All these electrochemical features are over imposed to the $\mathrm{Li}-\mathrm{C}$ reversible storage processes, which are evidenced as monotonous curves in Fig. 7 and as very broad differential profiles in Fig. 8.

\subsection{Comparison of Sn-PMCMT and PMCMT differential profiles}

Figs. 9a-c shows a comparison between the differential profiles of PMCMT and Sn-PMCMT anodes during cycles 1, 10, and 50, respectively. Even if the morphology and chemistry of the carbon matrix in PMCMT and Sn-PMCMT powders can be only scarcely reproducible, the superimposition of the differential profiles of the

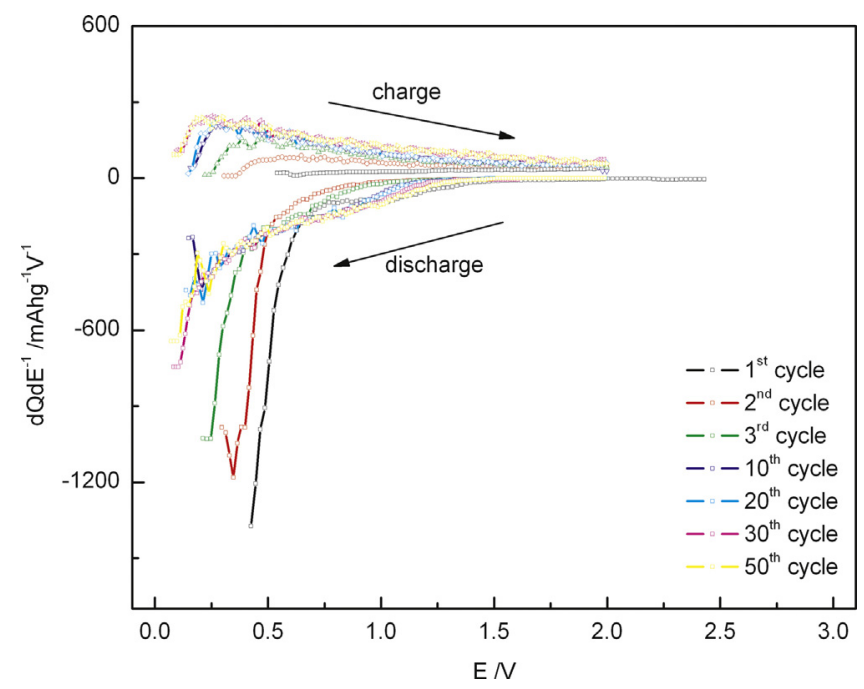

Fig. 8. Calculated $d Q d E^{-1}$ vs. $E$ differential profiles of pristine PMCMT anode. $T=20^{\circ} \mathrm{C}$; limiting capacity $=200 \mathrm{mAh}^{-1}$ (cycles $1,2,3,10$, and 20), $250 \mathrm{mAh}^{-1}$ (cycles 30 and 50); specific currents $=200 \mathrm{mAg}^{-1}$. two anodes is useful to discriminate the charge/discharge processes due to $\mathrm{Li}-\mathrm{Sn}$ alloying and $\mathrm{Li}$ storage by carbon matrix. Some relevant information can be gathered, in agreement with the results already shown, namely: (i) the carbon matrix utilization is comparable for both electrodes, since the low potential cut-offs are very close (and at the 50th cycle practically coincident); (ii) the sharp peaks, which describe Li storage processes occurring by welldefined phase transitions, are only related to Li-Sn alloying; (iii) the very broad and smooth profiles, which describe Li storage and release by processes involving amorphous phases, are due both to $\mathrm{Li}-\mathrm{Sn}$ and $\mathrm{Li}-\mathrm{C}$ reversible processes. This confirms that, while some of the capacity is due to the formation of $\mathrm{Li}-\mathrm{Sn}$ phases with welldefined stoichiometry and structure, a relevant amount of the $\mathrm{Li}-\mathrm{Sn}$ processes involve amorphous structures or the coexistence of several phases, leading to the overlapping of electrochemical signals. This phenomenon is emphasized by the sluggish kinetics shown as the cyclations proceed, leading to profiles as those evidenced in Fig. 8c (cycle 50) where no peaks can be detected, but only broad signals related to $\mathrm{Li}-\mathrm{Sn}$ (and $\mathrm{Li}-\mathrm{C}$ ) alloying and de-alloying.

The peaks related to the main $\mathrm{Li}-\mathrm{Sn}$ alloying and dealloying reactions have been assigned according to Refs. [13,23] and are marked in Fig. 9b as $(a-c)$ and $(d-g)$, respectively. The hysteresis between alloying and dealloying is typical of Li-Sn alloy electrodes $[23,24]$ and may be ascribed to different kinetics for the forward and backward processes. However, the anodic broad peak (h) at about $1.2 \mathrm{~V}$, that had been previously assigned to a kinetically hindered $\mathrm{Li}$ release from amorphous carbon [13,20], is present only in the differential profile of Sn-PMCMT anode, while it is absent in the case of pristine PMCMT. This suggests that it could be in turn assigned to the dealloying of residual amounts of $\mathrm{Li}$ from an amorphous $\mathrm{Li}-\mathrm{Sn}$ phase. The $\mathrm{Li}-\mathrm{C}$ processes should thus be assigned only to features marked as (i) and (j) and to the background of the profiles.

\subsection{Electrochemical impedance spectroscopy (EIS)}

The good performance obtained by the Sn-PMCMT anode, while being mainly due to the Sn contribution to the anode capacity by reversible Li-Sn alloying, could also be justified, at least in part, by a more efficient Li intercalation ability of the carbon matrix, this latter determined by the presence of finely dispersed nanosize Sn. In fact, the Sn metal nanoparticles can exhibit a catalytic role in aiding the desolvation of $\mathrm{Li}^{+}$'ad-ions' at electrode/electrolyte interface [29-34], thus enhancing Li insertion rate and reducing the polarization related to charge-transfer process. This is in agreement with previous findings on the positive effect of dispersed or layered Sn (and more generally of metals) toward the interfacial kinetics of graphite anodes $[8,9,35,36]$. In order to confirm this hypothesis, electrochemical impedance spectroscopy has been performed on both Sn-PMCMT and pristine PMCMT anodes, at a potential of $0.1 \mathrm{~V}$, where almost all $\mathrm{Li}-\mathrm{C}$ processes usually take place. Fig. 11 shows the overlapping of the Nyquist plots, normalized for the sake of comparison by the active mass of the electrodes.

In the middle-to-low frequency region, for both anodes, a $45^{\circ}$ line, which bends to a vertical one at the lowest frequency limit, can be associated to a diffusion in presence of a blocking electrode. In the case of pristine PMCMT anode, a further feature is roughly evidenced at intermediate frequencies, which reveals, in this case, that the diffusion takes place through porous, heterogeneous media [37-41]. A high-frequency feature, commonly describing $\mathrm{Li}^{+}$migration through a conductive passivation layer, is, for both electrodes, overlapped by a relevant middle-to-high frequency semicircle that describes the charge-transfer process (the characteristic frequency is about $60 \mathrm{~Hz}$ for both anodes). The resistance associated with the charge-transfer process is estimated to be of the order of $15 \Omega \mathrm{mg}^{-1}$ for the Sn-PMCMT anode as contrasted 


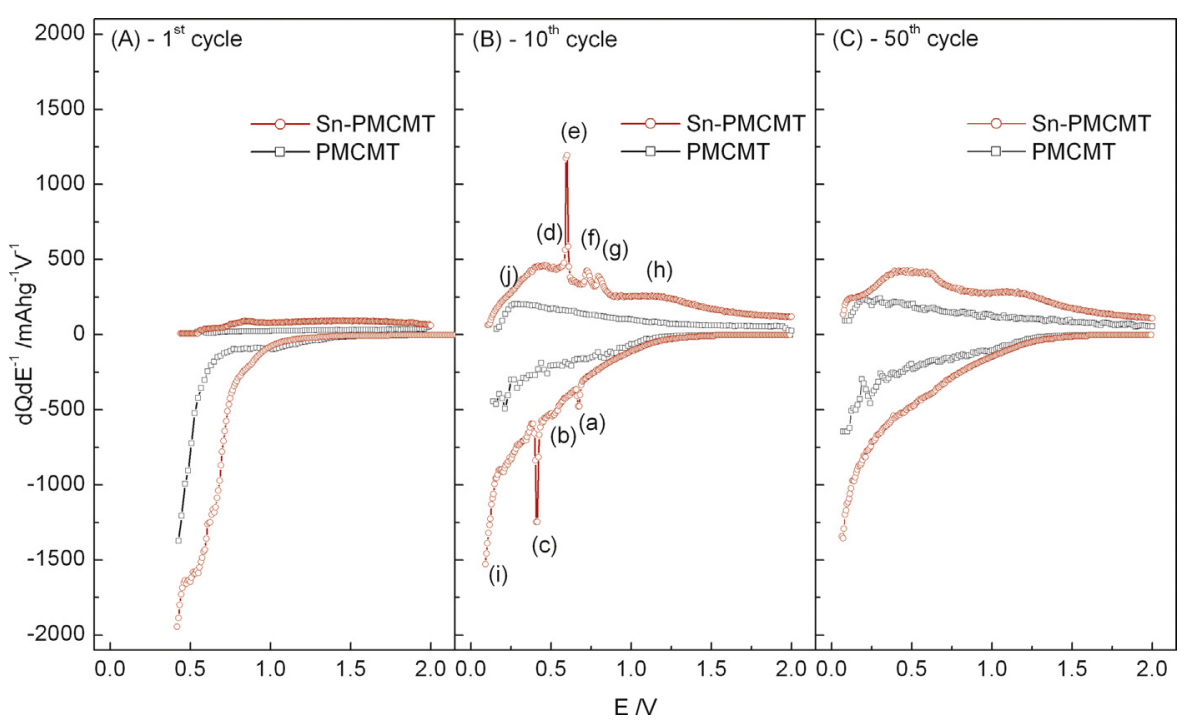

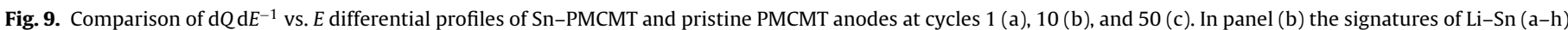
and $\mathrm{Li}-\mathrm{C}$ ( $\mathrm{i}$ and $\mathrm{j}$ ) reversible processes are detailed.

by $30 \Omega \mathrm{mg}^{-1}$ for the pristine PMCMT, which seems to confirm a catalytic action of the encapsulated Sn nano-powder toward the $\mathrm{Li}^{+}$ion de-solvation step at carbon/electrolyte interface [8]. It follows that the catalysis of the charge-transfer process is very effective in determining the good Sn-PMCMT performance at low temperature, where polarization phenomena could otherwise be a serious limitation to charge-discharge capacity $[8,9,35,36]$.

\subsection{Rate- and temperature-dependent charge/discharge behavior of Sn-PMCMT anode}

After having evidenced and explained the superior Sn-PMCMT anode performance, its electrochemical behavior has been evaluated in different and demanding operating conditions, as the case for very low temperatures.

To this aim, the Sn-PMCMT anode was subject to further galvanostatic cyclations in the temperature range $20^{\circ} \mathrm{C}$ to $-20^{\circ} \mathrm{C}$

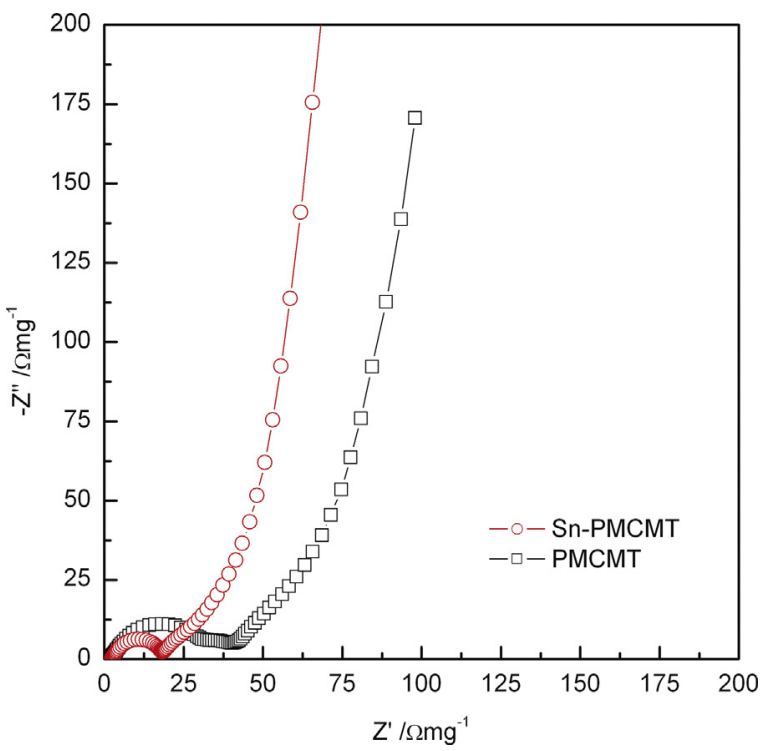

Fig. 10. Nyquist plots of Sn-PMCMT and pristine PMCMT anodes. $E=0.1 \mathrm{~V}$. $1 \mathrm{mHz}<f<100 \mathrm{kHz}$. and at specific currents of $125 \mathrm{~mA} \mathrm{~g}^{-1}, 250 \mathrm{mAg}^{-1}, 500 \mathrm{mAg}^{-1}$, corresponding approximately to $\mathrm{C} / 4, \mathrm{C} / 2,1 \mathrm{C}$ charge/discharge rates. The temperature- and rate-dependent charge/discharge behavior is summarized in Fig. 10.

The removal of the limitation in the capacity makes the anode active material fully accessible. In this condition, during the initial cycles a capacity increase up to about $850 \mathrm{mAh} \mathrm{g}^{-1}$ takes place. This capacity is the sum of contributions from reversible and irreversible processes. The latter, associated with low-potential irreversible $\mathrm{Li}-\mathrm{C}$ reactions, which are now accessible because of the removal of capacity limit, fade in few cycles and cannot further contribute to capacity. At the same time, the full anode utilization leads to an increase in the volume variations associated with the reversible $\mathrm{Li}-\mathrm{Sn}$ alloying, inducing a mechanical instability of the electrode (even if the $C$ matrix morphology can act as a buffer, as demonstrated in Ref. [13]). As a consequence, from the 205th cycle the reversible capacity starts to fade reaching a value of about $615 \mathrm{mAh} \mathrm{g}^{-1}$ at 210 th cycle. When the charge/discharge rate is raised, the increasing cell polarization

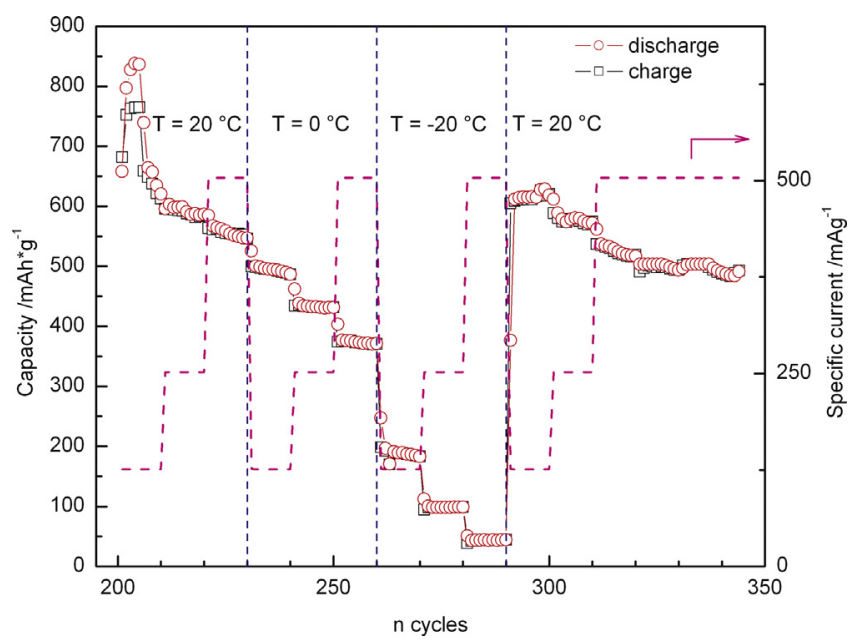

Fig. 11. Charge/discharge capacities and applied specific currents of Sn-PMCMT anode. $0.01<E<2 \mathrm{~V} . T=20^{\circ} \mathrm{C}$ (cycles 201-230 and 291-340), $0^{\circ} \mathrm{C}$ (cycles 231-260), $-20^{\circ} \mathrm{C}$ (cycles 271-290). 
forces the electrode to reach the set potential limit $(0.01 \mathrm{~V})$ before the Li storage reversible processes are completed. As a consequence, a slight decrease in capacity is observed $\left(590 \mathrm{mAh} \mathrm{g}^{-1}\right.$ at $\sim \mathrm{C} / 2=250 \mathrm{mAg}^{-1}, 550 \mathrm{mAh} \mathrm{g}^{-1}$ at $\sim 1 \mathrm{C}=500 \mathrm{mAg}^{-1}$ ).

Worth noticing here is that, after the fading of the capacity, shown immediately after the removal of the capacity limit, the subsequent charge/discharge cycles exhibit very stable performance. This behavior can be explained by admitting two concurrent phenomena. First, the increase of the polarization, due to the raise of charge/discharge rate, partly hinders the formation of the endmembers of $\mathrm{Li}-\mathrm{C}$ and $\mathrm{Li}-\mathrm{Sn}$ structures. These latter are known to be detrimental for electrode stability, because of the Li-Sn volume variations. In addition, it should be considered that the possible fragmentation of the particles with loss of contact, due to the large low-potential expansion/contraction of Li-Sn lattice, is confined to the initial cycles and limited to a small fraction of the active material present outside the carbon microtubes. Indeed, the porous carbon matrix appears to be able to buffer the volume variation phenomenon by embedding the majority of active material grains, giving outstanding mechanical stability to the composite anode [13].

When the temperature is lowered, the polarization of the cell is increased and the anode utilization decreases, resulting in lower charge/discharge capacities. Nevertheless, Coulombic efficiency still remains close to unity at all the current and temperature conditions investigated, confirming that, after the initial cycles in which some of the electrode reversibility is lost due to minor mechanical failure, the electrode morphology can efficiently buffer the volume variations associated with $\mathrm{Li}-\mathrm{Sn}$ alloying/dealloying. Reversible capacities of about $490 \mathrm{mAh} \mathrm{g}^{-1}, 430 \mathrm{mAh} \mathrm{g}^{-1}, 375 \mathrm{mAh} \mathrm{g}^{-1}$ are delivered at a temperature of $0^{\circ} \mathrm{C}$ and $\mathrm{C}$-rates of $\mathrm{C} / 4, \mathrm{C} / 2,1 \mathrm{C}$, while at $T=-20^{\circ} \mathrm{C}$ they become $190 \mathrm{mAh} \mathrm{g}^{-1}, 100 \mathrm{mAh} \mathrm{g}^{-1}, 45 \mathrm{mAh} \mathrm{g}^{-1}$ and $\mathrm{C} / 4, \mathrm{C} / 2,1 \mathrm{C}$, respectively.

When the cycling temperature is set again to $T=20^{\circ} \mathrm{C}$ (from cycle 291), the pristine charge/discharge capacity is restored, confirming the ability of the carbon-embedded Sn nanoparticles in buffering the Li-Sn volume variations, thus enhancing electrode stability. In fact, values of capacity close to the initial ones are obtained, i.e. about $610 \mathrm{mAh} \mathrm{g}^{-1}, 580 \mathrm{mAh} \mathrm{g}^{-1}, 500 \mathrm{mAh} \mathrm{g}^{-1}$ at specific current of $125 \mathrm{mAg}^{-1}$ (C/4), $250 \mathrm{mAg}^{-1}$ (C/2), $500 \mathrm{mAg}^{-1}$ (1C), respectively. These values compare, and more often outperform, the performances commonly reported in literature for similar Sn/C composite anode $[11,24,42]$.

\section{Conclusions}

The Li storage behavior of a Sn-PMCMT composite anode has been investigated by galvanostatic cyclations performed at different operating conditions. The application of a limitation in the delivered capacity has allowed to buffer the electrode stability issues, by distributing the large volume variations associated with Li-Sn alloying over several 'activation cycles' and by forcing the anode to operate relatively far from the Li deposition potential.

At the same time, reversible and irreversible $\mathrm{Li}-\mathrm{C}$ and $\mathrm{Li}-\mathrm{Sn}$ processes have been characterized. The two kinds of processes have been identified by comparison of the charge/discharge profiles, obtained with Sn-PMCMT and pristine PMCMT anodes cycled in similar conditions. It has been also shown, by ac-impedance analysis that the enhancement of $\mathrm{Li}-\mathrm{C}$ intercalation kinetics seems to play a role in enhancing the performance of the Sn-PMCMT anode.

Furthermore, the behavior of the enhanced anode has been investigated at several charge/discharge rates and temperatures, revealing that even at the lowest temperature studied the electrode can deliver relevant amounts of capacity.
Finally, it has been demonstrated that when the temperature is restored to $20^{\circ} \mathrm{C}$ the Sn-PMCMT anode can deliver capacity values of the order of about $500 \mathrm{mAh} \mathrm{g}^{-1}$ at $1 \mathrm{C}$ rate after more than 340 cycles. These figures outperform those commonly reported in literature for $\mathrm{Sn} / \mathrm{C}$ composite anodes of similar morphology. This behavior confirms that $\mathrm{Sn}$ encapsulation by the PMCMT matrix can efficiently buffer volume variations and mechanical issues related to $\mathrm{Li}-\mathrm{Sn}$ reversible alloying.

\section{Acknowledgments}

The researches leading to these results have received funding from the European Union Seventh Framework Programme (FP7/2007-2013) under grant agreement no. 265644, Project "APPLES Advanced, High Performance, Polymer Lithium Batteries for Electrochemical Storage".

\section{References}

[1] V. Etacheri, R. Marom, R. Elazari, G. Salitra, D. Aurbach, Challenges in the development of advanced Li-ion batteries: a review, Energy \& Environmental Science 4 (2011) 3243

[2] J.-M. Tarascon, Key challenges in future Li-battery research, Philosophical Transactions of the Royal Society A 368 (2010) 3227.

[3] F. Croce, M.L. Focarete, J. Hassoun, I. Meschini, B. Scrosati, A safe, high-rate and high-energy polymer lithium-ion battery based on gelled membranes prepared by electrospinning, Energy \& Environmental Science 4 (2011) 921

[4] O. Chusid, Y. Ein-Eli, M. Babai, Y. Carmeli, D. Aurbach, Electrochemical and spectroscopic studies of carbon electrodes in lithium battery electrolyte systems, Journal of Power Sources 43 (1993) 47.

[5] D. Aurbach, Y. Ein-Eli, O. Chusid, M. Babai, Y. Carmeli, H. Yamin, The correlation between the surface chemistry and the performance of Li-carbon intercalation anodes for rechargeable 'Rocking-Chair' type batteries, Journal of the Electrochemical Society 141 (1994) 603.

[6] D. Aurbach, Y. Ein-Eli, B. Markovsky, The dependence of the performance of Li-C intercalation anodes for Li-ion secondary batteries on the electrolyte solution composition, Electrochimica Acta 39 (1994) 2559.

[7] D. Aurbach, Y. Ein-Eli, B. Markovsky, A. Zaban, S. Luski, Y. Carmeli, H. Yamin, The study of electrolyte solutions based on ethylene and diethyl carbonates for rechargeable Li batteries: II. Graphite electrodes, Journal of the Electrochemical Society 142 (1995) 2882.

[8] F. Nobili, M. Mancini, S. Dsoke, R. Tossici, R. Marassi, Low-temperature behavior of graphite-tin composite anodes for Li-ion batteries, Journal of Power Sources 195 (2010) 7090

[9] F. Nobili, S. Dsoke, T. Mecozzi, R. Marassi, Metal-oxidized graphite composite electrodes for lithium-ion batteries, Electrochimica Acta 51 (2005) 536.

[10] H. Mori, Percolation structure, in: M. Hosokawa, K. Nagi, M. Naito, T. Yokoyama (Eds.), Nanoparticles Technology Handbook, 1st ed., Elsevier, Amsterdam, 2007, p. 206 (Ch. 4).

[11] M. Winter, J.O. Besenhard, M.E. Spahr, P. Novak, Insertion electrode materials for rechargeable lithium batteries, Advanced Materials 10 (1998) 725.

[12] N.A. Kaskhedikar, J. Maier, Lithium storage in carbon nanostructures, Advanced Materials 21 (2009) 2664.

[13] I. Meschini, F. Nobili, M. Mancini, R. Marassi, R. Tossici, A. Savoini, M.L. Focarete, F. Croce, High-performance Sn@carbon nanocomposite anode for lithium batteries, Journal of Power Sources 226 (2013) 241

[14] J. Li, R.B. Lewis, J.R. Dahn, Sodium carboxymethyl cellulose. A potential binder for Si negative electrodes for Li-ion batteries, Electrochemical and Solid State Letters 10 (2007) A17.

[15] B. Lestriez, S. Bahri, I. Sandu, L. Roué, D. Guyomard, On the binding mechanism of CMC in Si negative electrodes for Li-ion batteries, Electrochemistry Communications 9 (2007) 2801.

[16] M. Mancini, F. Nobili, R. Tossici, M. Wohlfahrt-Mehrens, R. Marassi, High performance, environmentally friendly and low cost anodes for lithium-ion battery based on $\mathrm{TiO}_{2}$ anatase and water soluble binder carboxymethyl cellulose, Journal of Power Sources 196 (2011) 9665.

[17] M. Mancini, F. Nobili, R. Tossici, R. Marassi, Study of the electrochemical behavior at low temperatures of green anodes for Lithium Ion batteries prepared with anatase $\mathrm{TiO}_{2}$ and water soluble sodium carboxymethyl cellulose binder, Electrochimica Acta 85 (2012) 566.

[18] M.C. Smart, B.V. Ratnakumar, S. Surampudi, Use of organic esters as cosolvents in electrolytes for Lithium-ion batteries with improved low temperature performance, Journal of the Electrochemical Society 149 (2002) A361.

[19] H.-C. Shiao, D. Chua, H. Lin, S. Slane, M. Salomon, Low temperature electrolytes for Li-ion PVDF cells, Journal of Power Sources 87 (2000) 167.

[20] T. Zheng, W.R. McKinnon, J.R. Dahn, Hysteresis during lithium insertion in hydrogen-containing carbons, Journal of the Electrochemical Society 143 (1996) 2137.

[21] Y. Yu, L. Gu, C. Zhu, P.A. van Aken, J. Maier, Tin nanoparticles encapsulated in porous multichannel carbon microtubes: preparation by single-nozzle 
electrospinning and application as anode material for high-performance Libased batteries, Journal of the American Chemical Society 131 (2009) 15984.

[22] Y. Yu, L. Gu, C. Wang, A. Dhanabalan, P.A. van Aken, J. Maier, Encapsulation of Sn@carbon nanoparticles in bamboo-like hollow carbon nanofibers as an anode material in lithium-based batteries, Angewandte Chemie International Edition 48 (2009) 6485.

[23] Y.S. Jung, K.T. Lee, J.H. Ryu, D. Im, S.M. Oh, Sn-carbon core-shell powder for anode in lithium secondary batteries, Journal of the Electrochemical Society 152 (2007) A1452.

[24] J. Hassoun, G. Derrien, S. Panero, B. Scrosati, A nanostructured Sn-C composite lithium battery electrode with unique stability and high electrochemical performance, Advanced Materials 20 (2008) 3169.

[25] L.Y.Beaulieu, S.D. Beattie, T.D. Hatchard, J.R. Dahn, The electrochemical reaction of lithium with tin studied by in situ AFM, Journal of the Electrochemical Society 150 (2003) A419.

[26] R.A. Huggins, Lithium alloy negative electrodes, Journal of Power Sources 81-82 (1999) 13.

[27] J.R. Dahn, T. Zheng, Y. Liu, J.S. Xue, Mechanisms for lithium insertion in carbonaceous materials, Science 270 (1995) 590.

[28] M. Winter, K.-C. Moeller, J.O. Besenhard, Electrochemical lithium storage in non-graphitic carbons, in: G.-A. Nazri, G. Pistoia (Eds.), Lithium Batteries. Science and Technology, Kluwer, New York, 2004, p. 160, Ch. 5.

[29] P.G. Bruce, M.Y. Saidi, The mechanism of electrointercalation, Journal of Electroanalytical Chemistry 322 (1992) 93

[30] K. Xu, Y. Lam, S.S. Zhang, T.R. Jow, T.B. Curtis, Solvation sheath of Li+ in nonaqueous electrolytes and its implication of graphite/electrolyte interface chemistry, Journal of Physical Chemistry C 111 (2007) 7411.

[31] K. Xu, "Charge-transfer" process at graphite/electrolyte interface and the solvation sheath structure of $\mathrm{Li}^{+}$in nonaqueous electrolytes, Journal of the Electrochemical Society 154 (2007) A162.

[32] T. Abe, H. Fukuda, Y. Iriyama, Z. Ogumi, Solvated Li-ion transfer at interface between graphite and electrolyte, Journal of the Electrochemical Society 151 (2004) A1120.
[33] T. Abe, F. Sagane, M. Ohtsuka, Y. Iriyama, Z. Ogumi, Lithium-ion transfer at the interface between lithium-ion conductive ceramic electrolyte and liquid electrolyte-a key to enhancing the rate capability of Lithium-ion batteries, Journal of the Electrochemical Society 152 (2005) A2151.

[34] K. Xu, A. von Cresce, U. Lee, Differentiating contributions to "ion transfer" barrier from interphasial resistance and Li desolvation at electrolyte/graphite interface, Langmuir 26 (2010) 11538.

[35] M. Mancini, F. Nobili, S. Dsoke, F. D’Amico, R. Tossici, F. Croce, R. Marassi, Lithium intercalation and interfacial kinetics of composite anodes formed by oxidized graphite and copper, Journal of Power Sources 190 (2009) 141.

[36] F. Nobili, M. Mancini, P.E. Stallworth, F. Croce, S.G. Greenbaum, R. Marassi, Tincoated graphite electrodes as composite anodes for Li-ion batteries. Effects of tin coatings thickness toward intercalation behavior, Journal of Power Sources 198 (2012) 243.

[37] D. Aurbach, M. Levi, E. Levi, H. Teller, B. Markovsky, G. Salitra, U. Heider, L. Heider, Common electroanalytical behavior of Li intercalation processes into graphite and transition metal oxides, Journal of the Electrochemical Society 145 (1998) 3024.

[38] J.P. Meyers, M. Doyle, R.M. Darling, J. Newman, The impedance response of a porous electrode composed of intercalation particles, Journal of the Electrochemical Society 147 (2000) 2930

[39] M.D. Levi, D. Aurbach, Impedance of a single intercalation particle and of nonhomogeneous, multilayered porous composite electrodes for Li-ion batteries, Journal of Physical Chemistry B 108 (2004) 11693.

[40] M.D. Levi, D. Aurbach, Simultaneous measurements and modeling of the electrochemical impedance and the cyclic voltammetric characteristics of graphite electrodes doped with lithium, Journal of Physical Chemistry B 101 (1997) 4630

[41] F. Nobili, S. Dsoke, M. Mancini, R. Tossici, R. Marassi, Electrochemical investigation of polarization phenomena and intercalation kinetics of oxidized graphite electrodes coated with evaporated metal layers, Journal of Power Sources 180 (2008) 845 ,

[42] N. Pereira, L.C. Klein, G.G. Amatucci, Particle size and multiphase effects on cycling stability using tin-based materials, Solid State Ionics 167 (2004) 29. 